\title{
On Grasping a Tumbling Debris Object with a Free-Flying Robot
}

\author{
Roberto Lampariello* \\ * Robotics and Mechatronics Center, German Aerospace Center \\ (DLR), Oberpfaffenhofen, D-82234 Wessling, Germany \\ (e-mail:Roberto.Lampariello@dlr.de)
}

\begin{abstract}
The grasping and stabilization of a tumbling, non-cooperative target satellite by means of a free-flying robot is a challenging control problem, which has been addressed in increasing degree of complexity since 20 years. A novel method for computing robot trajectories for grasping a tumbling target is presented. The problem is solved as a motion planning problem with nonlinear optimization. The resulting solution includes a first maneuver of the Servicer satellite which carries the robot arm, taking account of typical satellite control inputs. An analysis of the characteristics of the motion of a grasping point on a tumbling body is used to motivate this grasping method, which is argued to be useful for grasping targets of larger size.
\end{abstract}

Keywords: Trajectory planning, Autonomous control, Space robotics

\section{INTRODUCTION}

Active Debris Removal (ADR) measures are being recognized more and more among the Space Agencies and other parties worldwide as a necessary step in the advance of space technologies, due to the rising hazard which existing debris, especially in Low Earth Orbit (LEO), is presenting. Experts in the field of Space Debris have recently provided alarming evidence that the debris population in LEO will grow in time solely due to sporadic collisions between the uncontrolled objects which are currently in orbit (1). It is also argued that a substantial removal action, precisely five objects to be removed per year, is necessary, in order to at least keep the current debris population constant in size (1). At stake are the whole economical and scientific assets in orbit, with their immense impact on our society (e.g., Earth observation, climate measurements, tsunami warnings, satellite navigation, etcetera), which would be highly at risk of serious damage in an environment filled with debris fragments.

A suitable technology to address ADR is orbital robotics: a Servicer satellite equipped with a suitable rendezvous $\&$ docking capacity and with a robot manipulator, apt to perform a grasping task of typical tumbling debris objects. There exists already a large amount of literature which addresses the control of such so-called free-flying robots specifically for the grasping task (see next Section). Orbital demonstration missions are even now being worked upon around the world (e.g. DEOS in Germany (2), Phoenix in the US (3), to mention two), which partly stem from precursor, related orbital robotics missions such as ROTEX (4), ETS-VII (5), ROKVISS (4) or Orbital Express (6) and others (7), and which will become reality in the coming years. One aspect which still needs some attention within this context is the understanding of the challenges which arise from the nature of the tumbling motion of the target objects which are to be grasped. Debris objects in orbit generally consist of satellites which

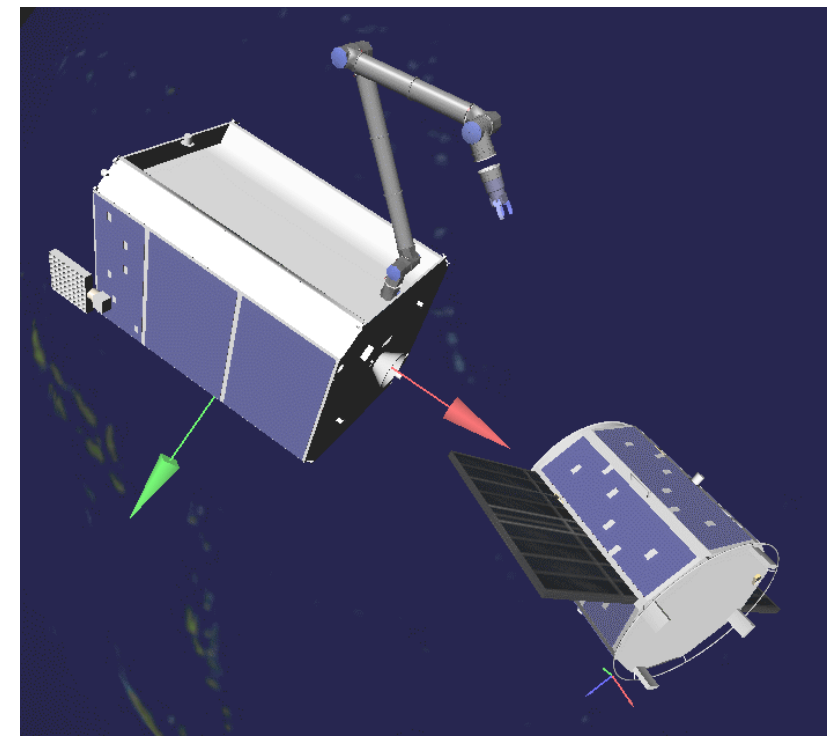

Fig. 1. Orbital scenario: Servicer satellite with $7 \mathrm{DOF}$ manipulator and Target satellite with solar panels. Orbital coordinate system shown (x - V-bar: red; $\mathrm{y}$ - out of orbital plane: green. One grasping point coordinate system on Target shown.

have reached their end-of-life or have suffered from some malfunction, or of last stages of rocket launchers. These objects are generally in an uncontrolled tumbling state. Note that still today there is no empirical evidence to support one or the other conjecture with regards to the expected tumbling motion of a given target. Theoretically, one can argue in favor of a slow flat-spin motion (rotation about a fixed axis of rotation), resulting from internal energy dissipation, or of a stable orientation brought about the gravity gradient torque (8). However, collisions with meteoroids, or with other debris objects, as well as other dynamic effects (see the chaotic tumbling of Saturn's 
aspherical moon Hyperion (9)), do not allow making any concluding statements.

In this paper, the characteristics of the dynamics of a tumbling target is first addressed, with specific attention to the motion of a selected grasping point on it. Then, based on the grasping method first described in (10), a more general grasping strategy is presented, which takes account of the challenges which result from the tumbling dynamics, where these are: being able to reach the grasping point at all from the chosen position of the Servicer satellite, while avoiding collisions with appendages on the target object; being able to do so in a time when a communication link to ground exists, since this allows an operator's supervisory role and as such, relaxes the necessity of onboard autonomy. These problems are also dependent on the target size and on the type of communication link, which is either direct or through a geostationary relay satellite.

A new formulation of the motion planning method used for solving the grasping problem is also described which provides a better computational efficiency with respect to that to be found in (10). Results are presented for the simulation scenario shown in Fig. 1.

The paper is then structured as follows: a bibliography is first provided in subsection 1.1 and a problem formulation is presented in subsection 1.2. Section 2 addresses the motion of the grasping point on a tumbling target. Section 3 addresses the grasping task details. Section 4 provides results and section 5 gives the conclusions.

\subsection{Related literature}

The grasping problem was treated in (10) as a trajectory planning problem (open loop control), and was solved with nonlinear optimization. The related target motion prediction and tracking control tasks were first addressed in (11) and (12) respectively. The planning task at hand results in a highly nonlinear, constrained optimization problem, to account for the typical robot box constraints, for the free-floating multibody dynamics, but also for collision avoidance, which is particularly important due to the possible presence of appendages (solar panels, antennas, etc.) on the debris object.

It is argued here that local control methods (13) (14) are bound to fail in general conditions, if they do not make use of a feasible reference trajectory, which includes a specification of the relative position between the Servicer and the target to be grasped. It is in fact evident that there is no simple measure to determine whether the grasping point is reachable from the current configuration (accounting for robot workspace limitations, also affected by the dynamic coupling with the satellite base) and whether the trajectory which will derive from a local control law will be feasible at all times (collision avoidance with appendages, sustainability of necessary actuation forces during the stabilization phase which follows grasping, kinematic and dynamic singularities, etcetera).

A great deal of the work to be found in the literature on the space robot grasping task, which covers a time span of over 15 years, is based on nonlinear feedback control and therefore is prone to suffer from the problems deriving from locality mentioned above. There are also many papers on motion control for free-floating and free-flying robots (see review (15)), of which however very few treat the collision avoidance issue. In (16), the trajectory generation of the approach phase was addressed, while treating the chaser satellite with robot as a point mass and the target as a rotating rigid body with a large span (to account for the solar panels). It is argued that the major danger is the potential for collision between the satellite and the robot. Optimization is used to find safe kinematical trajectories, while optimizing a safety metric, based on the time to impact in case of robot control failure, as well as fuel expenditure and time.

\subsection{Problem statement}

The sequence of events for the grasping and stabilization of a tumbling debris object, or Target, by means of a satellite equipped with a robot manipulator, or Servicer, may be described as follows (10):

- the Servicer is at first in an observation position, called the Observation point (see Fig. 6);

- Servicer Approach: the Servicer approaches its grasping position, called Mating point, by means of its actuation (see Fig. 7);

- Robot Approach: the robot manipulator on the Servicer performs a maneuver to bring its end-effector in the vicinity of the grasping point on the tumbling Target;

- Robot Tracking: the robot end-effector tracks the grasping point for a few seconds with subsequent homing-in and closing of the grasp;

- Robot Stabilization: the relative motion between the Servicer and the Target is stabilized with the robot;

- Compound stabilization: finally, the motion of the Servicer-Target compound is stabilized by means of the Servicer actuators.

Note that the Servicer is only actuated in the first approach and in the last compound stabilization phases and presents a free-floating dynamics in between. The Robot Tracking phase is introduced to minimize the impact between the robot and the Target at the grasp. The Servicer Approach phase is necessary to guarantee that the grasping point is in reach of the robot.

The problem we address here is that of providing a feasible Servicer-Robot trajectory to execute the grasping task, based on the sequence above. The target trajectory is assumed to be determined by a motion estimation and prediction algorithm (e.g., as in (11)), which processes stereo-camera or LIDAR images. The motion prediction is then fed to the motion planner to compute a feasible grasping reference trajectory. We will consider the scenario shown in Fig. 1, which presents a target satellite with solar appendages. The method that is proposed here should however be extendable to larger targets.

We will assume that the tumbling Target presents only one useful grasping point, for the purpose of our analysis. In fact, finding a suitable structure to grasp is a recognized problem (17). It is also assumed that the grasping point is predefined by an operator, to relax the degree of autonomy of the proposed method. 
A communication link to ground is assumed to be necessary during the grasping operation, to upload the reference trajectory to the robot, which must be computed on ground due to the limited computational power on board. It is also considered to be useful for supervising the grasping operation (or even perform the task in shared autonomy (4)). Two possible communication links to ground are possible: through a relay satellite in geostationary orbit or through a direct link to ground. While in the first case, a link can be assumed possible for at least a half-orbital period, for the second the time-of-contact typically has a duration of eight to ten minutes. The execution of the complete grasping task should be possible within this time.

The tumbling motion is assumed to be general, in terms of the satellite orientation (due to the reasons described in Section 1) and limited between $+/-4 \mathrm{deg}$./sec. for the angular velocity in the three Cartesian directions. The latter value may stem from a limitation of the computer vision algorithms, which are assumed to run through the communication link on ground (4).

\section{MOTION OF A GRASPING POINT ON A TUMBLING TARGET}

As stated in the previous subsection, we assume that the Target only presents one grasping point. This may be a little stringent - think of a rocket upper stage, for which the adapter ring could present more than one grasping point. However, this choice is made for the purpose of analysis. Consider then the Target shown in Fig. 2. The figure also shows the orientation of the orbital reference frame (assumed inertial). The orientation of the satellite is initially aligned with the inertial frame.

The Target inertia is defined to be $\mathbf{I}=[50.300 ; 0105.18$ $0 ; 0 \quad 0 \quad 105.97] \mathrm{kg} \mathrm{m}^{2}$, which represents a realistic axialsymmetric satellite. The vector from the centre of mass to the grasping point is $\left[\begin{array}{lll}1.0 & 0.5 & 0.5\end{array}\right] \mathrm{m}$. Assigning the initial conditions for the relative angular velocity between the Servicer and the Target to be $[-2-4-2]$ deg./sec. and integrating its equations of motion for a simulation time of 5000 seconds (approximately one LEO orbit period), the trajectory traced by the grasping point in the $\mathrm{y}-\mathrm{z}$ plane is shown in Fig. 3.

The outcome is a trajectory which belongs to the surface of a spherical segment. This is no surprise, given that the so-

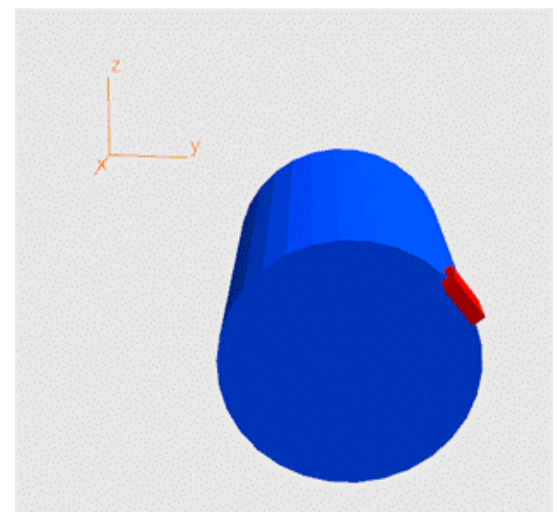

Fig. 2. Target satellite with one grasping point (red). Inertial frame shown.

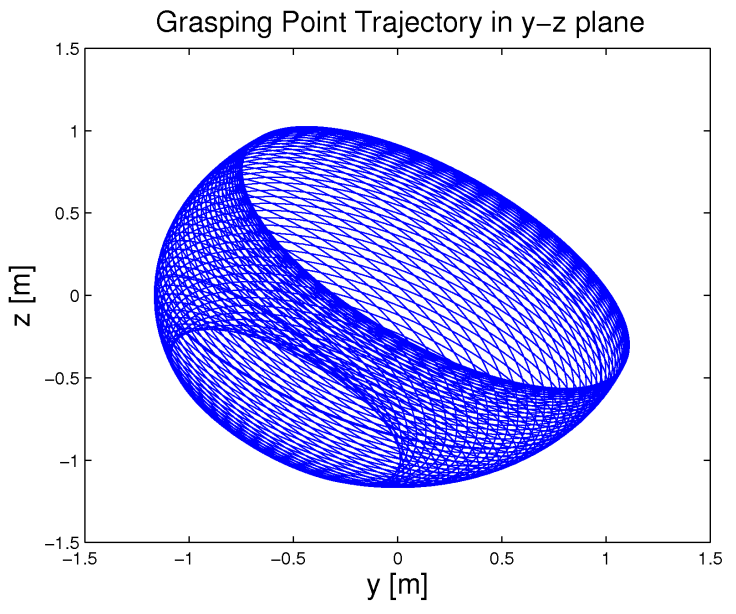

Fig. 3. Grasping point trajectory for initial angular velocity $[-2-4-2] \mathrm{deg} / \mathrm{sec}$

lution of a free rigid-body motion is such that the angular momentum is constant, thus providing a fixed direction in the inertial space, and the maximum (or minimum) principal axis of inertia describes a cone around it, of variable periodic aperture (therefore contained between a maximum and a minimum value) (8).

The first interesting point to note is that there are regions where the grasping point never enters. It is therefore necessary that the workspace of the robot overlaps with the spherical segment at the point in space and time that the grasping should take place. Furthermore, in the example given, there happen to be plenty of grasping opportunities, when we imagine ourselves positioned in front of the Target (e.g., coordinates $\left.\left[\begin{array}{lll}2 & 0 & 0\end{array}\right] \mathrm{m}\right)$, due to the fact that the chosen angular velocity results in many instances in which the grasping point is reachable in the given simulation time.

If we change the initial conditions of the angular velocity only slightly, say to $\left[\begin{array}{lll}-4 & -2 & -4\end{array}\right] \mathrm{deg}$./sec., we obtain, for the same simulation time, the trajectory shown in Fig. 4. The previous property of a limited spanned region is still

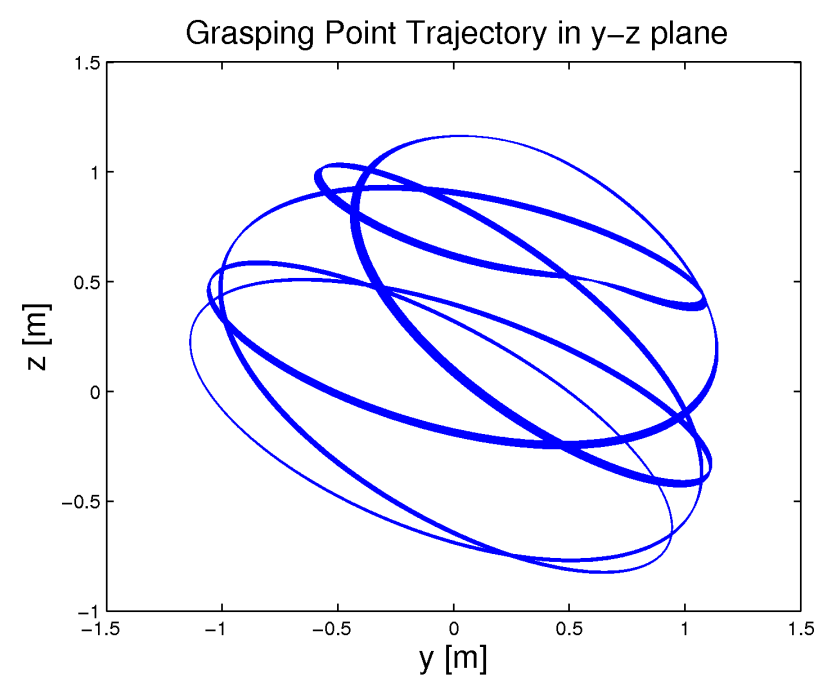

Fig. 4. Grasping point trajectory for initial angular velocity $[-4-2-4] \mathrm{deg} / \mathrm{sec}$ 
present of course. However, the shape of the trajectory has changed substantially, despite the small change in angular velocity. It can be shown that these results are also very sensitive to the inertial properties of the body.

Furthermore, nothing has been said about the orientation of the grasping point yet. In fact, the direction of the angular momentum vector is, as argued in Sec. 1, generic. As such, the shapes shown in Fig.s 3 and 4 will be oriented in inertial space accordingly. Furthermore, the orientation of the grasping point on the body is not represented in these figures, but varies along the trajectory. This is also an important element of the grasping task, since the endeffector has a limited relative orientation to the Target with which is can grasp it (see example in Sec. 4).

As a conclusion we can say that the position of the trajectory of the grasping point is generally confined to a limited region of unknown size and orientation and that it must be ensured that the robot workspace overlaps with this region for grasping. This in turn requires defining a suitable relative position between Servicer and Target. If appendages are present, collision avoidance needs to be taken into account in this procedure and parts of the region which contains the grasping point trajectory may therefore still not be accessible. Obviously, the larger the target, the stronger become these considerations (the recently lost ESA satellite Envisat is 26 meters long).

Finally, if we superimpose the effect of only having an 8 minute coverage for a given orbit, the result is shown in Fig. 5, with the grasping point trajectory during a direct link, i.e. for only 8 minutes. The opportunities to grasp are much fewer and the locations at which it is possible are spread apart. The same figure also shows the same information for a GEO-link with an equatorial LEO orbit, for which the coverage is the worst (i.e. half an orbital period). Although this has a clear advantage over a direct link, the reachability problem is still generally present. A GEO-link also presents the problem of having to deal with the intrinsic time delay of 600 to $800 \mathrm{~ms}$, which results from the round-trip-time of the signal. Such a delay may

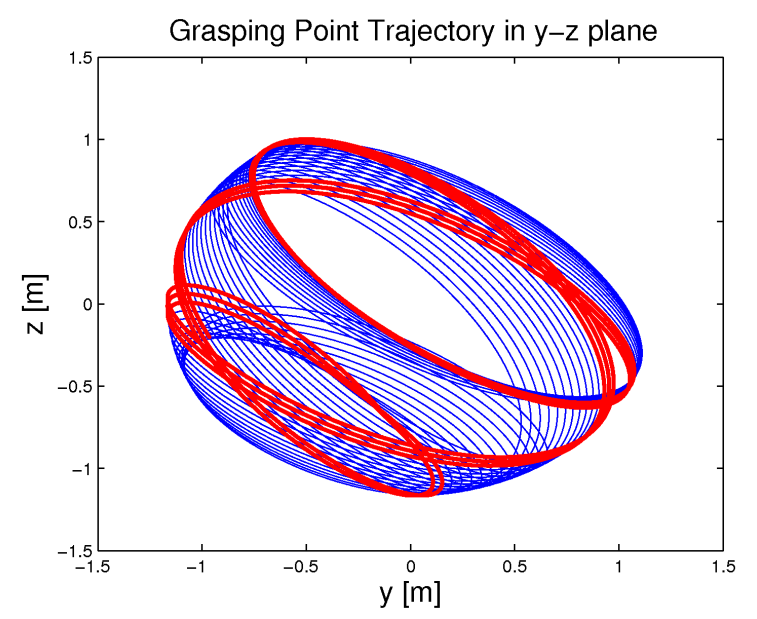

Fig. 5. Grasping point trajectory for initial angular velocity [-2 -4 -2] deg/sec and communication link coverage for half-orbit period (blue thin line) and 8 minutes (red thick line) be detrimental when grasping a tumbling Target, without appropriate means of motion extrapolation.

\section{METHOD FOR THE GRASPING TASK}

Two factors limit the planning of a feasible trajectory for the grasping task: the first is the limited communication time to ground, which results in a limited number of opportunities which can be used to perform the grasping, as described in Sec. 2; the second is the fact that the motion prediction algorithm used to support the motion planner (see Sec. 1.2), can only predict the Target motion for a limited time (in (11) the prediction time was 100 seconds). This limit results from the noisy data of the visual sensors (stereo-cameras or LIDAR) and the resulting uncertainty in the dynamic model used to propagate the tumbling motion.

The most general way to obviate this problem is to consider a maneuver of the Servicer to bring the robot in the vicinity the grasping point at the time when a communication to ground is possible and within the prediction time limit. This feature also allows to address the grasping problem for a large tumbling Target, for which the grasping point is bound to be initially out of reach.

\subsection{Motion planning problem}

The constrained optimization problem which results for the grasping task of interest was already described in (10). Its salient features are reported here for clarity. Extension to it are then presented, which lead to a better performance.

The motion planning problem is divided in (10) into two parts: the robot approach and tracking phases, followed by the robot stabilization phase (see Sec. 1.2). For the first phase, the robot joint positions are parameterized in time with polynomials or B-splines and two extra parameters are defined to parameterize the position of the Servicer (Vbar and R-bar) at the beginning of the robot maneuver in the orbital plane. For the second phase, which starts with the final motion conditions of the previous phase, only the joints positions are parameterized, again with polynomials or B-splines, to solve the stabilization problem, in which the velocity of the robot joints is brought to zero. Inequality nonlinear constraints are formulated for the joint positions, velocities and internal torques, as well as collision avoidance between all bodies of the satellites and robot. The method of solution is based on gradient-based optimization (SQP) and single shooting, which involves the integration of the equations of motion for every iteration of the optimization process.

The method was extended to include the following features:

Servicer point-to-point maneuver A Servicer point-topoint maneuver was integrated in the planning procedure. The resulting trajectory, with a trapezoidal profile in velocity, is collision free. A maximum velocity and maximum acceleration parameterize the Servicer trajectory, to account for realistic spacecraft AOCS dynamics. 
Initial guess with solution of inverse kinematics problem

The single-shooting method used to solve the optimization problem requires long computation times, since the equations of motion need to be solved via numerical integration for every iteration of the optimization process. Note that the integration must be performed with a high numerical accuracy to ensure that the angular momentum of the free-floating system remains constant throughout the integration. An alternative method was developed, in which the final position of the robot approach phase is determined by solving an inverse kinematics problem for the case in which the Servicer only has the two translational degrees of freedom in position in the orbital plane (therefore nine degrees of freedom in total). A solution to this problem provides a feasible configuration of the Servicer-Robot system which brings the robot end-effector in the desired position. This configuration is then joined to the predefined initial configuration via a polynomial or B-spline function, to obtain an initial guess for the original optimization problem.

\subsection{Time line of the grasping subtasks}

The resulting time sequence for the proposed method is as follows:

- 100 sec. Target motion observation time: the visual sensor data is recorded and transmitted to ground.

- 30 sec. computation time: the visual data is processed to produce the motion estimates and motion prediction of 225 seconds. This is fed to the motion planner which computes a feasible trajectory, if there is one.

- 200 sec.: spacecraft point-to-point maneuver. The duration of this maneuver is function of the allowed velocity and acceleration of the Servicer. 200 seconds is an adequate number for a Target of the size of the scenario considered here.

- 10 sec. robot approach maneuver

- 12,5 sec. robot tracking and stabilization maneuver

The resulting total time is 6 minutes.

\section{RESULTS}

An example solution is shown in Fig.s 6 to 8, in which the initial position of the Servicer in the Observation point, the position in the Mating Point and that of the robot after the robot stabilization are shown. The single grasping point is highlighted with a coordinate frame on the metal ring on the Target.

It is important to note in this example that the desired orientation of the end-effector with respect to the Target is parallel to the z-axis of the grasping point coordinate frame (blue in figure), as shown in Fig. 8. This is a condition imposed by the necessity to successively be able to perform a berthing task between the two satellites. This is also the reason why the Servicer moves to the Mating Point position shown in Fig. 7, from which this necessary relative orientation can be achieved. A time evolution of the Servicer maneuver and the necessary AOCS inputs can be found in Fig. 9, which shown the feasibility of the solution. Note however, that for this same performance, a grasping task for a large satellite could require very

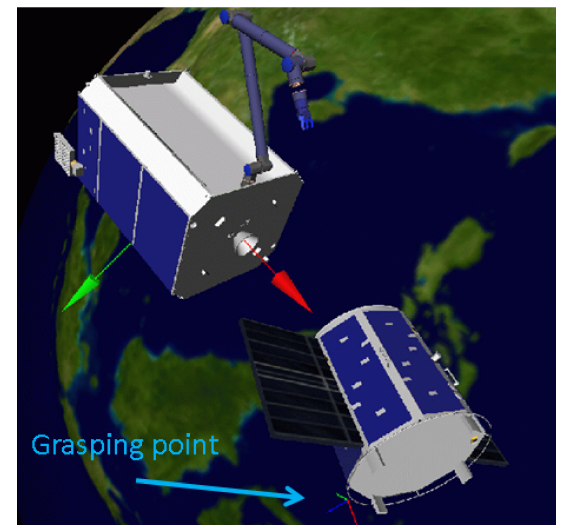

Fig. 6. Servicer in Observation point, origin of orbital frame

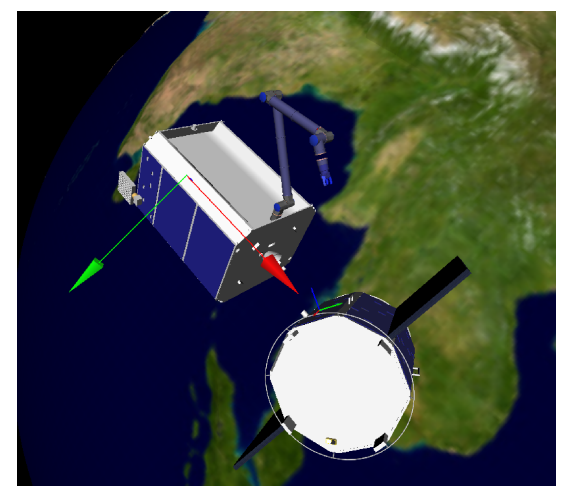

Fig. 7. Servicer in Mating point: [0.49 0 2.2] m

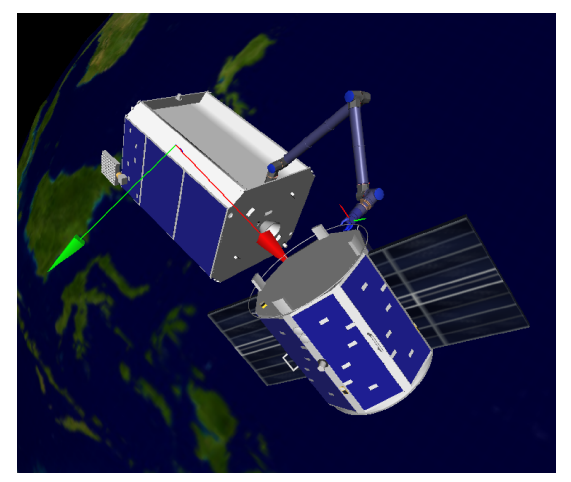

Fig. 8. End of robot stabilization phase

long times for the Servicer maneuver and therefore long prediction times.

Finally, of interest are also the internal forces and torques in the robot during the stabilization phase. The initial tumbling angular velocity in this case was [-2 -2 4 deg./sec.. The inertial properties of the Servicer and Target were taken to be: mass $_{\text {Servicer }}=685 \mathrm{~kg}, \mathbf{I}_{\text {Servicer }}=$ $\left[\begin{array}{lllllll}276.3 & 0 & 0 ; 0 & 496.9 & 0 ; 0 & 0 & 476.0\end{array}\right] \mathrm{kg} \mathrm{m} \mathrm{m}^{2}$, mass Target $=$

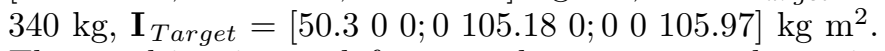
The resulting internal forces and torques are shown in Fig. 10 and 11. The plots show that all forces and torques are well within the structural limits of the robot. Note that for the torque these are never found to exceed $40 \mathrm{Nm}$ peak and that lower values could still be easily achieved by prolonging the stabilization phase, e.g. from 10.0 to 20.0 sec.. 

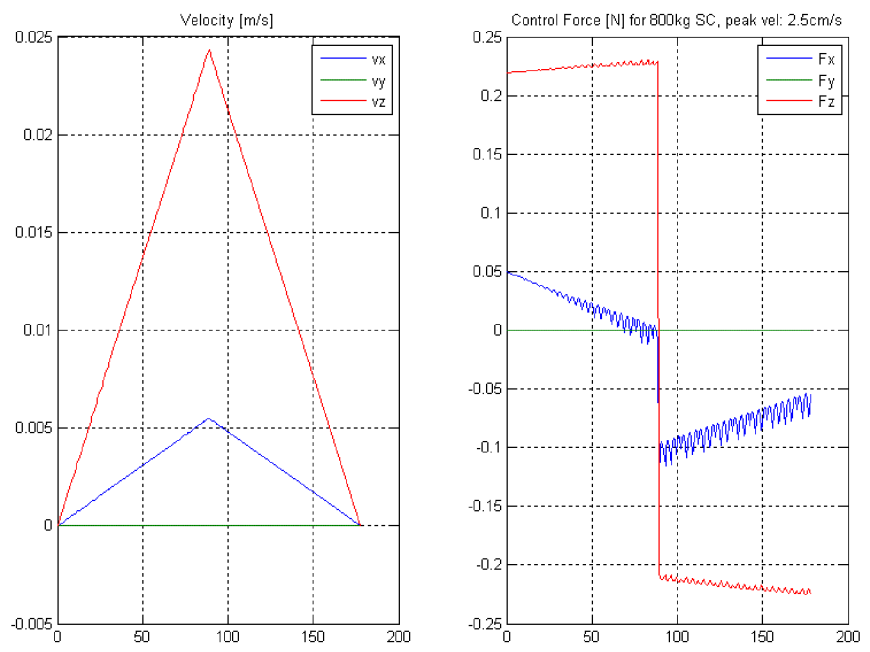

Fig. 9. Typical planned velocity profile of Servicer approach maneuver and equivalent actuation forces. Image provided by Ismar Masic, SpaceTech GmbH

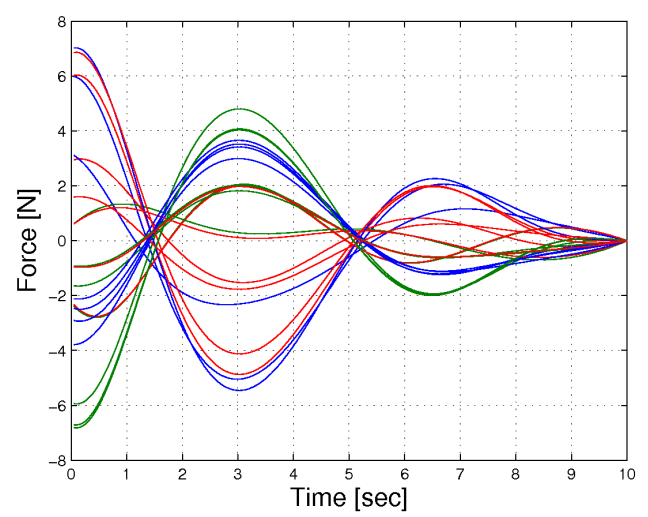

Fig. 10. Three internal force components for each of 7 robot joints during stabilization phase

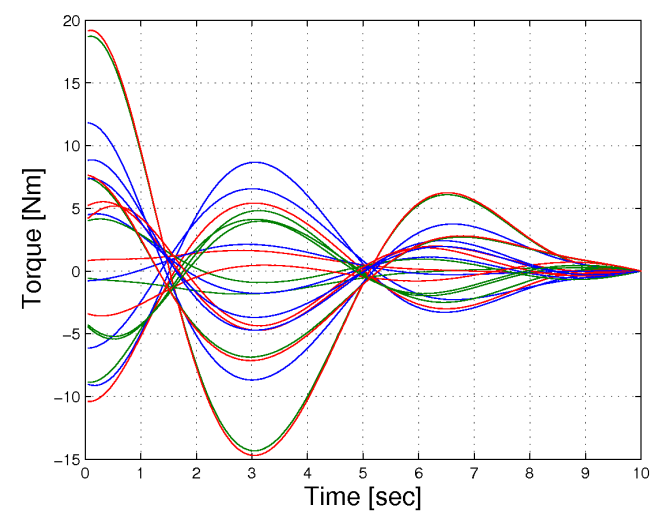

Fig. 11. Three internal torque components for each of 7 robot joints during stabilization phase

\section{CONCLUSION}

In addressing the grasping task of a tumbling Target by means of a Servicer satellite equipped with a manipulator arm, the necessity of moving the Servicer to an adequate position relative to the Target was underlined, by analyz- ing the characteristics of the typical motion of a grasping point on a tumbling rigid body and by accounting for the limited operational time available due to the limited communication time to ground. A motion planning approach was described which can deal with this necessity by providing a dynamically feasible and collision free trajectory of the Servicer, to be executed before the robot maneuver to grasp the Target. An open challenge was recognized for grasping large tumbling debris objects with the proposed method, for which the necessary prediction times may be very long. Further work will address this point.

\section{REFERENCES}

[1] Liou, J.-C., "An Updated Assessment of the Orbital Debris Environment in LEO," The Orbital Debris Quarterly News, vol. 14, no. 1, pp. 7-8, Jan. 2010.

[2] P. Rank, Q. Mühlbauer, W. Naumann, and K. Landzettel, "The DEOS Automation and Robotics Payload", in ASTRA, ESTEC, Holland, 2011.

[3] www.darpa.mil/our_work/tto/programs/ phoenix.aspx

[4] G. Hirzinger, B. Brunner, R. Lampariello, K. Landzettel, J. Schott, B.-M. Steinmetz, Advances in Orbital Robotics, ICRA2000, Vol. 1, pp. 898-907, 2000.

[5] Oda, M., "Summary of NASDA's ETS-VII robot satellite mission", Journal of Robotics and Mechatronics Vol.12, No.4, pp. 417-424, 2000.

[6] Kennedy, F.G., "Orbital Express: Accomplishments and Lessons Learned", Advances in the Astronautical Sciences, Vol. 131, Guidance and Control, 2008.

[7] K. Yoshida, "Achievements in space robotics", IEEE Robotics Automation Magazine, vol. 16, no. 4, 2009.

[8] Hughes, P., Spacecraft Attitude Dynamics, Dover Publication Inc., 1986.

[9] J. Wisdom and S.J. Peale, "Chaotic Rotation of Hyperion", ICARUS 58, 1987.

[10] Lampariello, R.: 'Motion Planning for the On-orbit Grasping of a Non-cooperative Target Satellite with Collision Avoidance', i-SAIRAS 2010, Japan, 2010.

[11] Hillenbrand, U., Lampariello, R.: 'Motion and Parameter Estimation of a Free-Floating Space Object from Range Data for Motion Prediction', i-SAIRAS 2005.

[12] Abiko, S. Lampariello, R. and Hirzinger, G.: 'Impedance Control for a Free-Floating Robot in the Grasping of a Tumbling Target with Parameter Uncertainty', IROS 06, Beijing, China, October 2006.

[13] Aghili, F.: "A Prediction and Motion-Planning Scheme for Visually Guided Robotic Capturing of FreeFloating Tumbling Objects With Uncertain Dynamics", IEEE Transactions on Robotics, Vol. 28, No.3, pp. 634-649, 2012.

[14] Yoshida, K., et al: "On the Capture of Tumbling Satellite by a Space Robot", IROS 06, China.

[15] Moosavian, S., Papadopoulos, E.: "Free-flying robots in space: an overview of dynamics modeling, planning and control, Robotica, Vol.25, pp. 537-547, 2007.

[16] Jacobsen, et al, "Planning of Safe Kinematic Trajectories for Free-Flying Robots Approaching an Uncontrolled Spinning Satellite" ASME 2002 Conference, Montreal, Canada, 2002.

[17] De Peuter, et al: "Satellite Servicing in GEO by Robotic Service Vehicle", ESA Bull. 78, 1993, pp 33-39. 